PROCEEDINGS OF THE

AMERICAN MATHEMATICAL SOCIETY

Volume 130, Number 5, Pages 1365-1370

S 0002-9939(01)06276-1

Article electronically published on September 19, 2001

\title{
FREDHOLMNESS AND INVERTIBILITY OF TOEPLITZ OPERATORS WITH MATRIX ALMOST PERIODIC SYMBOLS
}

\author{
LEIBA RODMAN, ILYA M. SPITKOVSKY, AND HUGO J. WOERDEMAN
}

(Communicated by Joseph A. Ball)

\begin{abstract}
We consider Toeplitz operators with symbols that are almost periodic matrix functions of several variables. It is shown that under certain conditions on the group generated by the Fourier support of the symbol, a Toeplitz operator is Fredholm if and only if it is invertible.
\end{abstract}

\section{Preliminaries AND MAin RESUlt}

Let $\left(A P^{k}\right)$ be the algebra of complex valued almost periodic functions of $k$ real variables, i.e., the closed subalgebra of $L^{\infty}\left(\mathbb{R}^{k}\right)$ (with respect to the standard Lebesgue measure) generated by all the functions $e_{\lambda}(t)=e^{i\langle\lambda, t\rangle}$, where $\lambda=\left(\lambda_{1}, \cdots, \lambda_{k}\right) \in \mathbb{R}^{k}$. Here the variable $t=\left(t_{1}, \cdots, t_{k}\right) \in \mathbb{R}^{k}$, and

$$
\langle\lambda, t\rangle=\sum_{j=1}^{k} \lambda_{j} t_{j}
$$

is the standard inner product of $\lambda$ and $t$. For every $f(t) \in\left(A P^{k}\right)$ its Fourier series is defined by the formal sum

$$
\sum_{\lambda} f_{\lambda} e^{i\langle\lambda, t\rangle}
$$

where

$$
f_{\lambda}=\lim _{T \rightarrow \infty} \frac{1}{(2 T)^{k}} \int_{[-T, T]^{k}} e^{-i\langle\lambda, t\rangle} f(t) d t, \lambda \in \mathbb{R}^{k},
$$

and the sum in (1.1) is taken over the set $\sigma(f)=\left\{\lambda \in \mathbb{R}^{k}: f_{\lambda} \neq 0\right\}$, called the spectrum of $f$. The spectrum of every $f \in\left(A P^{k}\right)$ is at most a countable set. Denote by $\Lambda(f)$ the smallest additive subgroup of $\mathbb{R}^{k}$ which contains $\sigma(f)$. The mean $M\{f\}$ of $f \in\left(A P^{k}\right)$ is defined by $M\{f\}=f_{0}=\lim _{T \rightarrow \infty} \frac{1}{(2 T)^{k}} \int_{[-T, T]^{k}} f(t) d t$. For the general theory of almost periodic functions of one and several variables we refer the reader to [4, 8, 9] and to Chapter 1 in [10].

Let $\Delta$ be a non-empty subset of $\mathbb{R}^{k}$. Denote

$$
\left(A P^{k}\right)_{\Delta}=\left\{f \in\left(A P^{k}\right): \sigma(f) \subseteq \Delta\right\} .
$$

Received by the editors October 28, 2000.

2000 Mathematics Subject Classification. Primary 47B35, 43A60.

Key words and phrases. Almost periodic functions, Toeplitz operators, Fredholmness.

The research of all three authors was partially supported by NSF grant DMS-9988579. 
If $\Delta$ is an additive subgroup of $\mathbb{R}^{k}$, then $\left(A P^{k}\right)_{\Delta}$ is a unital subalgebra of $\left(A P^{k}\right)$. We denote by $\left(A P^{k}\right)_{\Delta}^{n \times n}$ the set (algebra if $\Delta$ is a subgroup of $\mathbb{R}^{k}$ ) of $n \times n$ matrices with entries in $\left(A P^{k}\right)_{\Delta}$.

Introduce an inner product on $\left(A P^{k}\right)$ by the formula

$$
\langle f, g\rangle=M\left\{f g^{*}\right\}, f, g \in\left(A P^{k}\right) .
$$

The completion of $\left(A P^{k}\right)$ with respect to this inner product is called the Besikovitch space and is denoted by $\left(B^{k}\right)$. Thus $\left(B^{k}\right)$ is a Hilbert space. The elementary exponentials $e_{\lambda}(t), \lambda \in \mathbb{R}^{k}$, form an orthonormal basis in $\left(B^{k}\right)$. For a nonempty set $\Lambda \subseteq \mathbb{R}^{k}$, define the projection

$$
\Pi_{\Lambda}\left(\sum_{\lambda \in \sigma(f)} f_{\lambda} e_{\lambda}(t)\right)=\sum_{\lambda \in \sigma(f) \cap \Lambda} f_{\lambda} e_{\lambda}(t),
$$

where $f \in\left(A P^{k}\right)$. The projection $\Pi_{\Lambda}$ extends by continuity to the orthogonal projection (also denoted $\Pi_{\Lambda}$ ) on $\left(B^{k}\right)$. We denote by $\left(B^{k}\right)_{\Lambda}$ the range of $\Pi_{\Lambda}$, or, equivalently, the completion of $\left(A P^{k}\right)_{\Lambda}$ with respect to the inner product (1.4). The vector valued Besikovitch space $\left(B^{k}\right)^{n \times 1}$ consists of $n \times 1$ columns with components in $\left(B^{k}\right)$, with the standard Hilbert space structure. Similarly, $\left(B^{k}\right)_{\Lambda}^{n \times 1}$ is the Hilbert space of $n \times 1$ columns with components in $\left(B^{k}\right)_{\Lambda}$.

A subset $S$ of $\mathbb{R}^{k}$ is called a halfspace if it is closed under multiplication by nonnegative real numbers and addition, and has the properties that $\mathbb{R}^{k}=S \cup(-S)$ and $S \cap(-S)=\{0\}$. A standard example of a halfspace is given by

$E_{k}=\left\{\left(x_{1}, \cdots, x_{k}\right)^{T} \in \mathbb{R}^{k} \backslash\{0\}: x_{1}=x_{2}=\cdots=x_{j-1}=0, x_{j} \neq 0 \Rightarrow x_{j}>0\right\} \cup\{0\}$.

The vectors in $\mathbb{R}^{k}$ are understood as column vectors; the superscript ${ }^{T}$ denotes the transpose. Clearly, when $k=1$ the only halfspaces are $[0, \infty)\left(=E_{1}\right)$ and $(-\infty, 0]$. It turns out (see, for example, [12 for a proof) that a set $S \subset \mathbb{R}^{k}$ is a halfspace if and only if there exists a real invertible $k \times k$ matrix $A$ such that

$$
S=\left\{A x: x \in E_{k}\right\}
$$

A halfspace $S$ naturally induces a total order $>_{S}$ on $\mathbb{R}^{k}$ by the rule: $\lambda>_{S} \mu$ if and only if $\lambda-\mu \in S$ and $\lambda \neq \mu$.

Given $f \in\left(A P^{k}\right)^{n \times n}$ and a nonempty subset $\Omega \subseteq \mathbb{R}^{k}$, the Toeplitz operator $T_{f}(\Omega)$ is defined on $\left(B^{k}\right)_{\Omega}^{n \times 1}$ as follows:

$$
T_{f}(\Omega)(g)=\Pi_{\Omega}(f g), \quad g \in\left(A P^{k}\right)_{\Omega}^{n \times 1}
$$

the definition is extended by continuity to $g \in\left(B^{k}\right)_{\Omega}^{n \times 1}$. We will be interested in the case when $\Omega=\Lambda^{\prime} \cap S$, where $\Lambda^{\prime}$ is an additive subgroup of $\mathbb{R}^{k}$ which contains $\Lambda(f)$, and $S$ is a halfspace. Note that the cases $\Lambda^{\prime}=\Lambda$ and $\Lambda^{\prime}=\mathbb{R}^{k}$ are not excluded.

We state the main result of this paper.

Theorem 1.1. Let $f \in\left(A P^{k}\right)^{n \times n}$. Assume that the additive subgroup $\Lambda^{\prime} \supseteq \Lambda(f)$ of $\mathbb{R}^{k}$ and the halfspace $S$ are such that the following condition is satisfied:

(A) For every $\mu>_{S} 0, \mu \in \Lambda^{\prime}$, there exists a countable set $\lambda_{j} \in \Lambda^{\prime}, j=1,2, \cdots$, such that $\mu>_{S} \lambda_{j}>_{S} 0$ for all $j$, and $\lambda_{j}-\lambda_{k} \notin \Lambda(f)$ for all $j \neq k$.

Then the operator $T_{f}\left(\Lambda^{\prime} \cap S\right)$ is Fredholm if and only if it is invertible. 
Results in the spirit of Theorem 1.1 are known in the literature. It is proved in [3] that Toeplitz operators on $L_{2}\left(\mathbb{R}^{n}\right)$ with (suitably interpreted) almost periodic symbols are invertible if and only if they are Fredholm. A particular case of Theorem [1.1 (where $k=1$ and $\Lambda^{\prime}=\mathbb{R}$ ) was proved in [5] (see also [6]); another proof of this particular case using the theory of limit operators ([11, [1]) is given in [7] (in the equivalent language of Wiener-Hopf operators) and 22. The approach of [3] is based on a far-from-trivial verification of the fact that the $C^{*}$-algebra generated by Toeplitz operators under consideration does not contain non-zero compact operators, and on the following known and simple fact: $A C^{*}$-algebra $\mathcal{A}$ of linear bounded operators on a Hilbert space that contains I has only zero intersection with the ideal $\mathcal{K}$ of compact operators if and only if every Fredholm operator that belongs to $\mathcal{A}$ is invertible. For the reader's convenience, here is a short proof of this fact: Assume $\mathcal{A} \cap \mathcal{K}=\{0\}$, and let $X \in \mathcal{A}$ be Fredholm. The $\operatorname{limit}_{\lim _{\varepsilon \rightarrow 0} \varepsilon\left(X^{*} X+\varepsilon I\right)^{-1}}$ exists in $\mathcal{A}$ and coincides with the orthogonal projection on the kernel of $X$. By assumption, this orthogonal projection, being a finite rank operator, must be zero. Applying this argument to $X^{*}$, we conclude also that Range $X$ is the whole Hilbert space, i.e., $X$ is invertible. Conversely, assume that every Fredholm operator in $\mathcal{A}$ is invertible, and let $K \in \mathcal{A} \cap \mathcal{K}$. Then for every real $c$ the operator $I+c K K^{*} \in \mathcal{A}$ is Fredholm, hence invertible. It follows that $\sigma\left(K K^{*}\right)=\{0\}$, therefore $K=0$.

Note that if condition (A) is satisfied, then the factor-group $\Lambda^{\prime} / \Lambda(f)$ is infinite. However, the condition of the factor-group $\Lambda^{\prime} / \Lambda(f)$ being infinite does not imply condition (A), as the following example shows: Let $\Lambda^{\prime}=\mathbb{Z}^{2} \subset \mathbb{R}^{2}$ (here $\mathbb{Z}$ stands for the set of integers),

$$
\Lambda(f)=\left\{(0, m) \in \mathbb{Z}^{2}: m \in \mathbb{Z}\right\}, \quad S=\left\{\left(x_{1}, x_{2}\right) \in \mathbb{R}^{2}: x_{1}>0 \text { or } x_{1}=0, x_{2} \geq 0\right\} .
$$

In this example, for every element $(0, m) \in \Lambda^{\prime}$ such that $(0, m)>_{S} 0$ there exist only finitely many elements $\lambda \in \Lambda^{\prime}$ for which $(0, m)>_{S} \lambda>_{S} 0$. Here, e.g., the Toeplitz operator with (scalar valued) symbol $f\left(t_{1}, t_{2}\right)=e^{i t_{2}}$, considered as an operator on $\left(B^{2}\right)_{\mathbb{Z}^{2} \cap S}^{1 \times 1}$, is Fredholm but not invertible.

The following two corollaries are noteworthy:

Corollary 1.2. If $f \in\left(A P^{k}\right)^{n \times n}$ and $S \subset \mathbb{R}^{k}$ is a halfspace, then the Toeplitz operator $T_{f}(S)$ is Fredholm if and only if $T_{f}(S)$ is invertible.

For the proof observe that $\Lambda(f)$ is at most countable, whereas for every $\mu>_{S} 0$ the set

$$
\left\{\lambda \in \mathbb{R}^{k}: \mu>_{S} \lambda>_{S} 0\right\}
$$

is a continuum (as easily follows from (1.5)). It remains to apply Theorem 1.1 with $\Lambda^{\prime}=\mathbb{R}^{k}$.

A subgroup $\Lambda \subseteq \mathbb{R}^{k}$ is called divisible if

$$
\lambda \in \Lambda \quad \Longrightarrow \quad \frac{1}{n} \lambda \in \Lambda \quad \text { for every positive integer } n,
$$

and it is called discrete if there exists an open neighborhood $U$ of 0 in $\mathbb{R}^{k}$ such that $U \cap \Lambda=\{0\}$.

Corollary 1.3. Let $f \in\left(A P^{k}\right)^{n \times n}$ and let $S \subset \mathbb{R}^{k}$ be a halfspace. If $\Lambda(f)$ is discrete and $\Lambda^{\prime} \supset \Lambda(f)$ is divisible, then the operator $T_{f}\left(\Lambda^{\prime} \cap S\right)$ is Fredholm if and only if it is invertible.

In particular, $\Lambda(f)$ is discrete if $f$ is a periodic matrix function. 


\section{Proof of the main Result}

We start with a lemma.

Lemma 2.1. Let $T$ be a Fredholm linear bounded operator on the orthogonal sum of Hilbert spaces $\mathcal{H}=\bigoplus_{j \in J} \mathcal{H}_{j}$, where the index set $J$ is infinite. Assume that each $\mathcal{H}_{j}$ is $T$-invariant. Then there exist a finite subset $J_{0} \subset J$ and $\varepsilon>0$ such that for every $j \in J \backslash J_{0}$ the restriction $\left.T\right|_{\mathcal{H}_{j}}$ of $T$ to $\mathcal{H}_{j}$ is invertible and the inequality

$$
\left\|\left.T\right|_{\mathcal{H}_{j}} x\right\| \geq \varepsilon\|x\|, \quad x \in \mathcal{H}_{j},
$$

holds.

Proof. Denote $T_{j}=\left.T\right|_{\mathcal{H}_{j}}, j \in J$, and let $J_{1}$ be the set of such $j \in J$ that $\operatorname{dim} \operatorname{Ker} T_{j}>0$. Since $\operatorname{Ker} T=\bigoplus_{j \in J} \operatorname{Ker} T_{j}$, this set must be finite. A similar reasoning applied to adjoint operators shows that $J_{2}=\left\{j \in J: \operatorname{dim} \operatorname{Ker} T_{j}^{*}>0\right\}$ is finite as well. Let $J_{0}=J_{1} \cup J_{2}$, and represent $T$ as the orthogonal sum of the operators $\hat{T}=\bigoplus_{j \in J_{0}} T_{j}$ and $\tilde{T}=\bigoplus_{j \in J \backslash J_{0}} T_{j}$. Observe that $\operatorname{Ker} \tilde{T}=\operatorname{Ker} \tilde{T}^{*}=\{0\}$. The Fredholmness of $T$ implies the Fredholmness, and thus invertibility, of $\tilde{T}$. But then

$$
\|T x\|=\|\tilde{T} x\| \geq\left\|\tilde{T}^{-1}\right\|^{-1}\|x\|
$$

for all $x \in \bigoplus_{j \in J \backslash J_{0}} \mathcal{H}_{j}$. In particular,

$$
\left\|\left.T\right|_{\mathcal{H}_{j}} x\right\| \geq\left\|\tilde{T}^{-1}\right\|^{-1}\|x\|
$$

for $x \in \mathcal{H}_{j}$.

It is easy to derive a necessary and sufficient condition for $T$ to be Fredholm. It consists of (2.1) combined with Fredholmness of $T_{j}$ for all $j \in J_{0}$. However, we do not need this statement in what follows.

Proof of Theorem 1.1. Assume the hypotheses and notation of Theorem 1.1, and let $T_{f}\left(\Lambda^{\prime} \cap S\right)$ be a Fredholm operator. We show that the kernel of $T_{f}\left(\Lambda^{\prime} \cap S\right)$ is trivial. Let $\left\{\Omega_{j}\right\}_{j \in J}$ be the collection of all distinct cosets of $\Lambda^{\prime}$ by $\Lambda(f)$, indexed with an index set $J$. It is easy to see that $\left(B^{k}\right)_{\Omega_{j} \cap S}^{n \times 1}$ is $T_{f}\left(\Lambda^{\prime} \cap S\right)$-invariant, for every $j \in J$. Indeed, fix $j \in J$. For an elementary exponential $e_{\lambda} x$, where $\lambda \in \Omega_{j} \cap S$ and $x$ a non-zero vector in $\mathbb{C}^{n}$, we have

$$
\sigma\left(f e_{\lambda} x\right) \subseteq \sigma(f)+\lambda \subseteq \lambda+\Lambda(f)=\Omega_{j} .
$$

So $\sigma\left(\Pi_{\Lambda^{\prime} \cap S}\left(f e_{\lambda} x\right)\right) \subseteq \Omega_{j}$. But it is also clear that

$$
\sigma\left(\Pi_{\Lambda^{\prime} \cap S}\left(f e_{\lambda} x\right)\right) \subseteq S
$$

therefore

$$
\sigma\left(\Pi_{\Lambda^{\prime} \cap S}\left(f e_{\lambda} x\right)\right) \subseteq S \cap \Omega_{j} .
$$

Since the elementary exponentials $e_{\lambda} x$ (where $\lambda$ is arbitrary in $\Omega_{j} \cap S$ and $x$ is an arbitrary element of a fixed orthonormal basis in $\mathbb{C}^{n}$ ) form an orthonormal basis in $\left(B^{k}\right)_{\Omega_{j} \cap S}^{n \times 1}$, it follows that the subspace $\left(B^{k}\right)_{\Omega_{j} \cap S}^{n \times 1}$ is $T_{f}\left(\Lambda^{\prime} \cap S\right)$-invariant. We also have the orthogonal decomposition

$$
\left(B^{k}\right)_{\Lambda^{\prime} \cap S}^{n \times 1}=\bigoplus_{j \in J}\left(B^{k}\right)_{\Omega_{j} \cap S}^{n \times 1} .
$$


Therefore, by Lemma 2.1 there exist a finite set $J_{0} \subset J$ and $\varepsilon>0$ such that the restriction of $T_{f}\left(\Lambda^{\prime} \cap S\right)$ to $\left(B^{k}\right)_{\Omega_{j} \cap S}^{n \times 1}$, call it $T_{j}$, is invertible for $j \in J \backslash J_{0}$, and moreover

$$
\left\|T_{j} y\right\| \geq \varepsilon\|y\| \quad \text { for all } y \in\left(B^{k}\right)_{\Omega_{j} \cap S}^{n \times 1} \quad\left(j \in J \backslash J_{0}\right) .
$$

Now assume that

$$
\Pi_{\Lambda^{\prime} \cap S}\left(f g_{0}\right)=0
$$

for some $g_{0} \in\left(B^{k}\right)_{\Omega_{j_{0}} \cap S}^{n \times 1},\left\|g_{0}\right\|=1$. Let

$$
h=\Pi_{\Lambda^{\prime} \backslash S}\left(f g_{0}\right) .
$$

In the Fourier series for $h, \sum_{\nu \in \Lambda^{\prime} \backslash S} h_{\nu} e_{\nu}$, we have

$$
\sum_{\nu \in \Lambda^{\prime} \backslash S}\left\|h_{\nu}\right\|^{2}<\infty
$$

and therefore there exists a finite set $Q \subseteq \Lambda^{\prime} \backslash S$ such that

$$
\sum_{\nu \in \Lambda^{\prime} \backslash S, \nu \notin Q}\left\|h_{\nu}\right\|^{2}<\varepsilon^{2} .
$$

Let $\mu \in Q$ be the largest element in $Q$ (with respect to the total order induced by $S$ ); thus $\mu \geq_{S} q$ for every $q \in Q$. By condition (A), there is $\mu_{0} \in \Lambda^{\prime} \backslash S$ such that $\mu_{0}>_{S} \mu$. We then have

$$
\sum_{\nu \in \Lambda^{\prime} \backslash S,-\mu_{0}+\nu \in S}\left\|h_{\nu}\right\|^{2}<\varepsilon^{2} .
$$

In addition, by condition (A), we may select countably many elements $\lambda_{j} \in \Lambda^{\prime} \backslash S$, $j=1,2, \ldots$, such that $\lambda_{j}>_{S} \mu_{0}$ and $\lambda_{j}-\lambda_{k} \notin \Lambda(f)$ for all $j \neq k$. Let $g_{j}=e_{\lambda_{j}} g_{0}$. Since $\Pi_{\Lambda^{\prime} \cap S}\left(f g_{0}\right)=0$ we have

$$
\begin{aligned}
& T_{f}\left(\Lambda^{\prime} \cap S\right)\left(g_{j}\right)=\Pi_{\Lambda^{\prime} \cap S}\left(f g_{j}\right)=\Pi_{\Lambda^{\prime} \cap S}\left(e_{\lambda_{j}} f g_{0}\right) \\
& \quad=\Pi_{\Lambda^{\prime} \cap S}\left(e_{\lambda_{j}}\left(\Pi_{\Lambda^{\prime} \backslash S}\left(f g_{0}\right)\right)\right)=\Pi_{\Lambda^{\prime} \cap S}\left(e_{\lambda_{j}}\left(\sum_{\nu \in \Lambda^{\prime} \backslash S, \nu \geq \lambda_{j} \lambda_{j}} h_{\nu} e_{\nu}\right)\right) .
\end{aligned}
$$

But in view of (2.3)

$$
\left\|\sum_{\nu \in \Lambda^{\prime} \backslash S, \nu \geq S \lambda_{j}} h_{\nu} e_{\nu}\right\|<\varepsilon
$$

thus

$$
\left\|T_{f}\left(\Lambda^{\prime} \cap S\right)\left(g_{j}\right)\right\|<\varepsilon=\varepsilon\left\|g_{j}\right\|
$$

Since the elements $\lambda_{j}$ belong to different cosets of $\Lambda^{\prime}$ by $\Lambda(f)$, we obtain a contradiction with (2.2), according to which the inequality (2.4) is possible only for $j \in J_{0}$.

We have proved that $\operatorname{Ker} T_{f}\left(\Lambda^{\prime} \cap S\right)=\{0\}$. Denote by $\hat{f} \in\left(A P^{k}\right)^{n \times n}$ the matrix function such that $\hat{f}(t)$ is the conjugate transpose of the matrix $f(t)$, for every $t \in \mathbb{R}^{k}$. It is easy to see that $\Lambda(\hat{f})=\Lambda(f)$. Also, $\left(T_{f}\left(\Lambda^{\prime} \cap S\right)\right)^{*}=T_{\hat{f}}\left(\Lambda^{\prime} \cap S\right)$. Applying the already proved part of Theorem 1.1 to $\hat{f}$, we see that

$$
\text { Range } T_{f}\left(\Lambda^{\prime} \cap S\right)=\left(B^{k}\right)_{\Lambda^{\prime} \cap S}^{n \times 1} \text {. }
$$

The invertibility of $T_{f}\left(\Lambda^{\prime} \cap S\right)$ follows. 


\section{REFERENCES}

[1] A. Böttcher, Yu. I. Karlovich, and V. S. Rabinovich. The method of limit operators for onedimensional singular integrals with slowly oscillating data. J. Operator Theory, 43:171-198, 2000. MR 2001d:45004

[2] A. Böttcher, Yu. I. Karlovich, and I. M. Spitkovsky. Convolution Operators and Factorization of Almost Periodic Matrix Functions. In preparation.

[3] L. Coburn, R. D. Moyer, and I. M. Singer. $C^{*}$-algebras of almost periodic pseudo-differential operators. Acta Math., 130:279-307, 1973. MR 54:3495

[4] C. Corduneanu. Almost Periodic Functions. J. Wiley \& Sons, 1968. MR 58:2006

[5] Yu. I. Karlovich. Algebras of convolution type operators with discrete groups of shifts and oscillating coefficients. Doctoral dissertation, Mathematical Institute, Georgian Academy of Sciences, Tbilisi, 1991.

[6] Yu. I. Karlovich. On the Haseman problem. Demonstratio Math., 26:581-595, 1993. MR 95a:47048

[7] Yu. I. Karlovich and I. M. Spitkovsky. (Semi)-Fredholmness of convolution operators on the spaces of Bessel potentials. Operator Theory: Advances and Applications, 71:122-152, 1994. MR 95h:47034

[8] B. M. Levitan. Almost Periodic Functions. GITTL, Moscow, 1953. MR 15:700a

[9] B. M. Levitan and V. V. Zhikov. Almost Periodic Functions and Differential Equations. Cambridge University Press, 1982. MR 84g:34004

[10] A. A. Pankov. Bounded and Almost Periodic Solutions of Nonlinear Differential Operator Equations. Kluwer, Dordrecht/Boston/London, 1990. MR 92f:35002

[11] V. S. Rabinovich. Pseudodifferential operators with operator symbols: Local invertibility and limit operators. Linear Topological Spaces and Complex Analysis, 1:58-73, 1994. MR 96b:47057

[12] L. Rodman, I. M. Spitkovsky, and H. J. Woerdeman. Factorization of almost periodic matrix functions of several variables and Toeplitz operators. Operator Theory: Advances and Applications, 122 (H. Bart, I. Gohberg, A.C.M. Ran, eds.): 385-416, 2001.

Department of Mathematics, The College of William and Mary, P.O. Box 8795, WilliamsBurg, ViRginia 23187-8795

E-mail address: lxrodm@math.wm.edu

Department of Mathematics, The College of William and Mary, P.O. Box 8795, Williamsburg, Virginia 23187-8795

E-mail address: ilya@math.wm.edu

Department of Mathematics, The College of William and Mary, P.O. Box 8795, Williamsburg, Virginia 23187-8795

E-mail address: hugo@math.wm.edu 